\title{
Terahertz Performance of a GaN-Based Planar Nanochannel Device
}

\author{
K. Y. Xu, ${ }^{1}$ Y. N. Wang, ${ }^{1}$ C. J. Zheng, ${ }^{1}$ J. W. Xiong, ${ }^{1}$ and G. Wang ${ }^{2}$ \\ ${ }^{1}$ Laboratory of Quantum Engineering and Quantum Materials, School of Physics and Telecommunication Engineering, \\ South China Normal University, Guangzhou 510006, China \\ ${ }^{2}$ State Key Laboratory of Optoelectronic Materials and Technologies, Sun Yat-sen University, Guangzhou 510275, China \\ Correspondence should be addressed to K. Y. Xu; xuky@scnu.edu.cn and G. Wang; stswangg@mail.sysu.edu.cn
}

Received 13 January 2014; Revised 16 March 2014; Accepted 25 March 2014; Published 10 April 2014

Academic Editor: Sheng-Po Chang

Copyright (C) 2014 K. Y. Xu et al. This is an open access article distributed under the Creative Commons Attribution License, which permits unrestricted use, distribution, and reproduction in any medium, provided the original work is properly cited.

\begin{abstract}
Using a combined two-dimensional-three-dimensional (2D-3D) ensemble Monte Carlo (EMC) model, the performance of a planar nanochannel device is studied at the terahertz $(\mathrm{THz})$ region. The device is based on a GaN/AlGaN heterostructure in which a two-dimensional electron gas (2DEG) forms at the interface. Simulation results reveal that, at low working frequencies, the performance of the device is almost frequency independent. However, when the working frequency is higher than $0.5 \mathrm{THz}$, obvious enhancements in the device performance have been observed. The enhancements are characterized by two resonant peaks at frequencies of about $4 \mathrm{THz}$ and $8 \mathrm{THz}$. Also, the frequency-dependent performance exhibits nonmonotonicity. Further studies show that the performance enhancements can be attributed to the excitations of $2 \mathrm{D}$ plasma waves in the device, with the emergence of the above resonant peaks corresponding to the formation of standing plasma waves. Moreover, simulation results show that the device performance increases monotonically with signal amplitude, when the device is unbiased. However, when a DC bias is applied, the performance remains almost unchanged for large signals but is significantly enhanced for small signals. Therefore, the device performance shows a strong nonmonotonic dependence on signal amplitude, and its minimal value occurs when the signal amplitude is only about $\sqrt{2}$ times the DC bias.
\end{abstract}

\section{Introduction}

In the past few decades, continuous miniaturization of semiconductor devices has led to high-speed operations and large-scale integration of electronics. A single silicon chip can now contain more than a billion transistors and operate at frequencies higher than $1 \mathrm{GHz}$. The operation frequency can be further increased by utilizing a planar architecture. In planar devices, electrodes are connected to the sides of the active semiconductor layer, rather than being placed on its top and bottom surface, as in conventional multilayered vertical-structured devices. As a result, very low parasitic capacitances are obtainable, leading to very high operating speeds. Planar nanodevices based on 2DEGs in semiconductor heterostructures have been demonstrated to operate at tens of $\mathrm{GHz}$ or more [1-3]. In particular, self-switching diodes (SSDs) have been shown to possess zero threshold voltages and be able to work as high-frequency detectors. At room temperature, they can operate at frequencies up to $1.5 \mathrm{THz}$ [4] at room temperature and up to $2.5 \mathrm{THz}$ [5] at a temperature of $150 \mathrm{~K}$.

Apart from improving the operational frequency of the devices, planar architecture can also lead to some additional mechanisms. It has been shown that when the thickness of the conducting layer is lower than $10 \mathrm{~nm}, 2 \mathrm{D}$ plasmonic noise is dominant, resulting in a geometric dependence of noise characteristics [6]. Moreover, it has been proved that in a submicron field-effect transistor (FET) with asymmetric boundary conditions, 2D plasma waves can be excited simply by a DC current [7]. This discovery has prompted intensive investigations of nanometer FETs as THz sources and detectors, resulting in substantial progress in this field [8-13].

The physics of plasma wave based collective electron transport is vastly different from conventional individual (uncorrelated) electron transport. However, the studies so far have been mostly limited to plasma waves occurring 
in submicron FET structures. In this study, based on a combined 2D-3D EMC model [14], we will focus on the role of $2 \mathrm{D}$ plasma waves in improving the $\mathrm{THz}$ performance of a two-terminal nanometer device. The paper is structured as follows. In Section 2, the structure of the device is first introduced and then the combined 2D-3D EMC model is concisely described. In Section 3, the frequency-dependent performance of the device is studied under various conditions, and the plasma related features are analysed in detail. In Section 4, the conclusions of this work are presented.

\section{Device Structure and Numerical Model}

Figures 1(a) and 1(b) schematically show the top view and cross section of a nanochannel device. The device is based on a GaN/AlGaN heterostructure, in which a 2DEG is formed at the GaN/AlGaN interface with a carrier concentration of $8.0 \times 10^{12} \mathrm{~cm}^{-2}$ [15]. The two insulating trenches (rectangular grey areas in Figure 1(a)) are created by etching through the 2DEG layer. Therefore, electrons have to pass through the narrow channel between the two trenches in order to conduct current from the left electron reservoir to the right one. It is evident that while the device performance at low frequencies might be trivial, at the $\mathrm{THz}$ region, it would be quite complex because additional phenomena, such as plasma waves, would be dominant.

In this work, we employed a combined 2D-3D EMC model based on a semiclassical 2D EMC method selfconsistently coupled with 3D Poisson equations. This combined model is developed from our entirely 2D model, which has been successfully applied in earlier studies [16, 17]. It is noteworthy that a fully 3D EMC model has been developed to study three-terminal T-branch junctions (TBJs) with a top gate terminal $[18,19]$. Despite minimizing the need for parameter fitting and including the effect of electron transfers from the channel to other layers, the $3 \mathrm{D}$ model leads to almost the same results as those obtained from an entirely 2D model for GaAs-based devices [20]. There are two reasons for the above agreement between an entirely $2 \mathrm{D}$ model and a $3 \mathrm{D}$ model. The first is that the operating properties of TBJs are mainly determined by the 2DEG layer, which can be well described by an entirely $2 \mathrm{D}$ model. The other is that an artificially introduced side gate in the $2 \mathrm{DEG}$ layer can serve the function of TBJs' top gate. If the top gate cannot be modelled as a side gate, at least a combined 2D-3D model is needed.

As in an entirely 2D model, all the electrons in the combined model are assumed to be confined within the 2DEG layer. This means that the effect of electron transfers from the channel to other layers is ignored. As such, the 2D EMC method is sufficient to describe the microscopic behaviour of electrons in devices. However, the main advantage of the combined model lies in upgrading the 2D Poisson solver in the entirely $2 \mathrm{D}$ model to a $3 \mathrm{D}$ Poisson solver. This would enable the proper inclusion of electric-field couplings beyond the 2DEG layer, which can lead to the appearance of $2 \mathrm{D}$ plasma waves. In order to sufficiently include the 3D electricfield couplings, Poisson equations should be solved in a domain beyond the device's realistic structure. As shown in Figure 1(b), the GaN substrate and a region (air) above the device surface, both of height $1 \mu \mathrm{m}$, were included in the simulations. Insulating trenches were assumed to have vertical sidewalls. Moreover, the depth of all the insulting trenches was assumed to be $1030 \mathrm{~nm}$. This uniform-depth treatment can avoid the trench-depth effect [14]. Dielectric constants used in the simulations for Air, AlGaN, and GaN were $1,8.5$, and 8.9 , respectively. For convenience, the effect of surface states at the semiconductor-air interface was included by a simple constant charge model with negative charge density, $N_{S}=-0.8 \times 10^{12} \mathrm{~cm}^{-2}$ [21]. Of course, the use of an advanced surface charge model, such as the self-consistent charge model, would result in more accurate results, but it is time consuming, and the results have no qualitative difference [22]. Geometric parameters used in the simulations can be found in Figure 1(a). All simulations were performed at room temperature with the left terminal grounded. The Poisson equations were solved in $5 \mathrm{~nm} \times 5 \mathrm{~nm}$ meshes with a time step of $1 \mathrm{fs}$. More information about the model can be found in our recent work $[14,23]$.

\section{Simulation Results and Analysis}

Figure 2 shows the frequency-dependent performance of the device shown in Figure 1. In the simulations, the right terminal was biased with different $\mathrm{DC}$ voltages of $V_{\mathrm{DC}}=$ $2.0 \mathrm{~V}$ (red curve), $1.5 \mathrm{~V}$ (green curve), $1.0 \mathrm{~V}$ (navy blue curve), $0.5 \mathrm{~V}$ (pink curve), and $0.0 \mathrm{~V}$ (powder-blue curve). Then, a sinusoidal signal with an amplitude of $V_{\mathrm{AC}}=0.5 \mathrm{~V}$ was also applied, and the AC current $I_{\mathrm{AC}}$ through the nanochannel was recorded. For ease of comparison, all the data were normalized to those obtained at the lowest simulated frequency, that is, $0.1 \mathrm{THz}$. We find that the performance of the device biased with a voltage of $1.0 \mathrm{~V}$ remains unchanged at low frequency, while showing obvious enhancement (i.e., the normalized amplitude of $I_{\mathrm{AC}}$ is larger than 1) when the frequency is higher than $0.5 \mathrm{THz}$. The normalized amplitude increases with frequency and reaches its first maximal value at a frequency of about $4 \mathrm{THz}$. Subsequently, it dramatically reduces to a minimum at a frequency of about $6.7 \mathrm{THz}$ and then increases again to reach the second maximal value at a frequency of about $8 \mathrm{THz}$. Similar trends are observed for devices with higher DC bias (i.e., $V_{\mathrm{DC}}=1.5 \mathrm{~V}$ and $2.0 \mathrm{~V}$ ). Moreover, results show that the enhancement is higher when a higher DC bias is applied. For example, the performance of the device at a DC bias of $2.0 \mathrm{~V}$ can be enhanced to more than 2.5 times that at low frequencies (see the red curve in Figure 2). This happens at a frequency of about $4 \mathrm{THz}$, at which a maximum power of about $10 \mu \mathrm{W}$ can be reached. However, for the device with smaller DC bias (i.e., $V_{\mathrm{DC}}=0.5 \mathrm{~V}$ and $0.0 \mathrm{~V}$ ), no enhancement is observed, and the normalized amplitude monotonically reduces with frequency, as shown by the pink and powder-blue curves in Figure 2.

In order to identify the origin of the above performance enhancements, efforts are devoted to compare nonenhanced results obtained at a low frequency of $0.1 \mathrm{THz}$ with the enhanced ones obtained at a high frequency of 4.0 THz. During simulations, the time-dependent 2D-current-distribution 


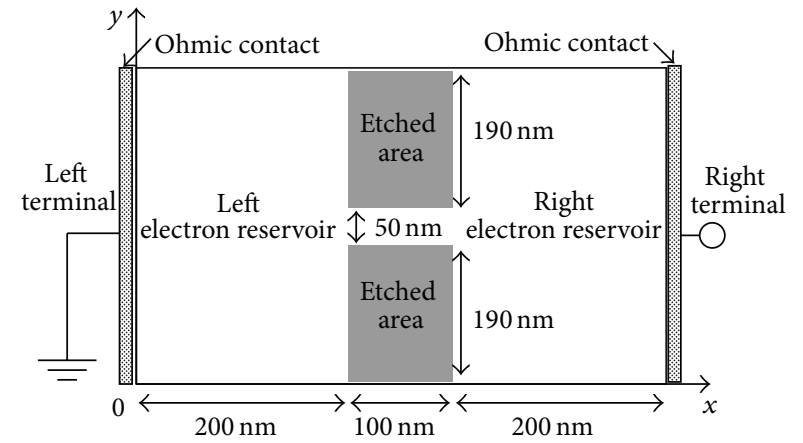

(a)

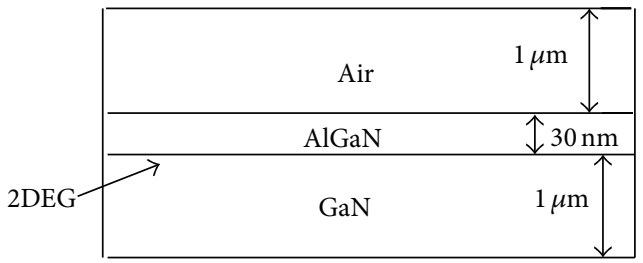

(b)

Figure 1: Schematic top view (a) and side view (b) of the simulated nanochannel device (not to scale). The grey areas and the white area in the top view represent insulating trenches and the 2DEG, respectively. A GaN/AlGaN interface is only $30 \mathrm{~nm}$ below the device surface, at which a sheet of 2DEG forms. In the simulations, all the insulating trenches are assumed to have vertical sidewalls and pass through the whole GaN/AlGaN heterostructure.

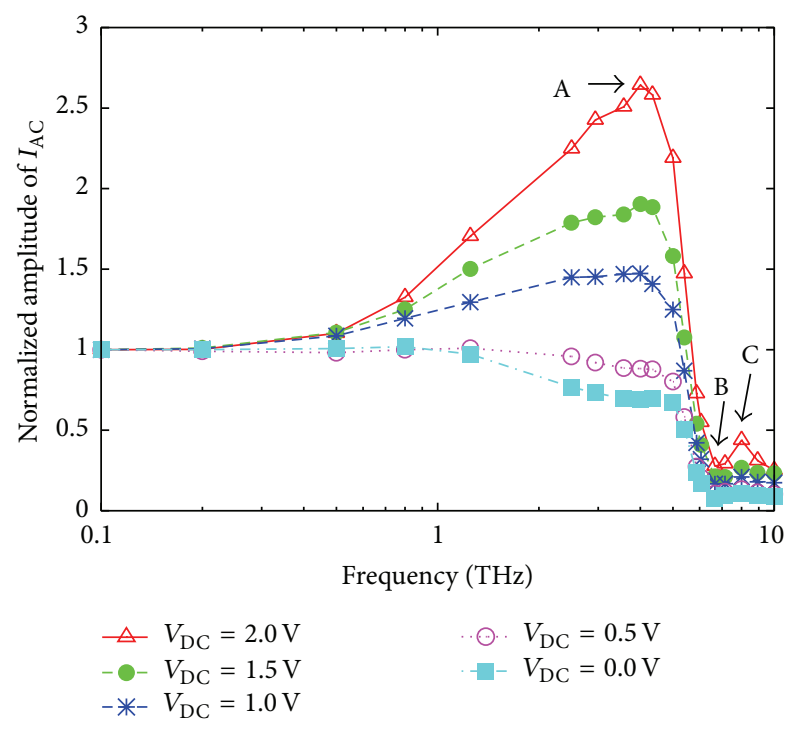

Figure 2: Frequency-dependent output-signal amplitude of the nanochannel device shown in Figure 1 when different DC voltages are applied (during all simulations, the input-signal amplitude was set to $0.5 \mathrm{~V})$.

$I(t, X, Y)$ in the entire device (including the channel and the two reservoirs) was recorded. Then, the recorded currents were summed along the $Y$ direction to obtain a timedependent $1 \mathrm{D}$-current-distribution $I(t, X)$ in the $X$ direction. Here, $t$ is the time, the $X$ direction is parallel to the channel, and the $Y$ direction is perpendicular to the channel, as shown in Figure 1. Results obtained at $0.1 \mathrm{THz}$ and at $4.0 \mathrm{THz}$ are shown in Figures 3(a) and 3(b), respectively. We find from Figure 3(a) that the low-frequency current distribution along the $X$ direction is uniform during the entire working period (i.e., the period of the applied signal) and synchronously changes with the applied signal. In contrast, the high-frequency results are quite nonuniform, because standing waves have formed in both reservoirs, as shown in
Figure 3(b). The emergence of standing waves in the device strongly suggests a possibility of plasma-wave excitations. The dispersion relations for 2D plasma waves are given by [6]

$$
f=\frac{1}{2 \pi} \sqrt{\frac{e^{2} n^{2 \mathrm{D}} k}{2 m_{0} m \varepsilon_{0} \varepsilon_{\mathrm{eff}}}},
$$

where $f$ and $k$ are the frequency and wave vector, respectively; $e$ is the electron charge; $n^{2 \mathrm{D}}=8 \times 10^{12} \mathrm{~cm}^{-2}$ is the $2 \mathrm{D}$ carrier concentration; $m_{0}$ and $m$ are the free and effective electron masses; $\varepsilon_{0}$ is the vacuum dielectric permittivity; and $\varepsilon_{\text {eff }}$ is the effective dielectric constant, which is defined as [24]

$$
\varepsilon_{\mathrm{eff}}=\frac{1}{2}\left[\varepsilon_{2}+\varepsilon_{1} \frac{1+\varepsilon_{1} \tanh (k d)}{\varepsilon_{1}+\tanh (k d)}\right] .
$$

In our case, $\varepsilon_{1}=8.5$ is the dielectric constant of AlGaN, $\varepsilon_{2}=8.9$ is the dielectric constant of GaN, and $d=30 \mathrm{~nm}$ is the thickness of the AlGaN layer. The standing wave conditions in the reservoirs are given by

$$
k=\frac{\pi(2 n-1)}{2 L}
$$

where $n=1,2,3 \ldots$ and $L=200 \mathrm{~nm}$ is the length of reservoirs. Substituting (2) and (3) into (1) and using the parameters provided above, one can observe that the frequency of standing waves is $4.5 \mathrm{THz}, 7.1 \mathrm{THz}$, and $8.5 \mathrm{THz}$ for $n=1,2$, and 3, respectively. Since damping effects (e.g., phonon scatterings) are not included in (1), analytic results obtained from (1)-(3) are expected to be higher than those obtained from the simulation. By making a comparison, one can find that each analytically obtained frequency is only a little higher than the frequency at special points $\mathrm{A}, \mathrm{B}$, and $\mathrm{C}$ shown in Figure 2. This confirms that the enhancement of device performance in the $\mathrm{THz}$ region is because of the excitations of 2D plasma waves. Moreover, the formation of standing plasma waves in the reservoirs is attributed to the performance peaks (special points A and C) shown in Figure 2. However, according to the analytic results, a peak, 


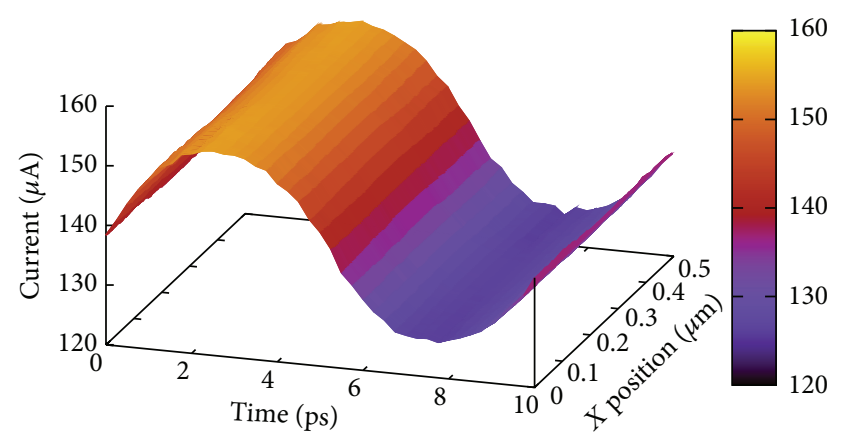

(a)

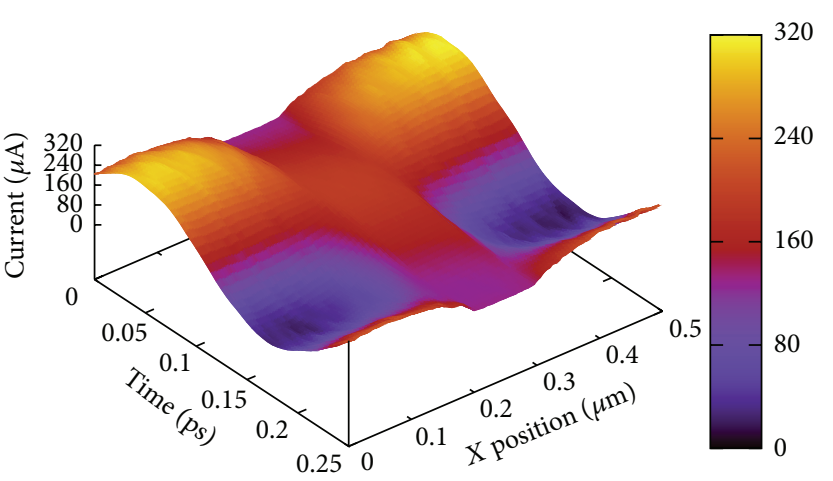

(b)

FIGURE 3: Time-dependent current distributions along the $X$ direction (defined in Figure 1(a)) for the device operating at the frequencies of $0.1 \mathrm{THz}$ (a) and $4.0 \mathrm{THz}$ (b).

rather than a valley, should emerge at point $\mathrm{B}$. To ascertain the reason for this anomaly, (1) was utilized to calculate the phase delay occurring in the plasma waves as they passed through the nanochannel. Considering that the electron density in the nanochannel is reduced by the surface states to about $75 \%$ of that in the reservoirs, a phase delay of $\pi$ can be obtained. Because of this $\pi$-phase delay, the standing waves in the two reservoirs have opposite effects on the device performance. As a result, the resonant peak at point $B$ disappears. From this calculation, we also find that the power-delay product for the device is frequency dependent.

It is well known that the length of electron reservoirs has negligible influence on the performance of traditional devices. However, as we have shown above, this is not true for nanodevices operating at the $\mathrm{THz}$ region, especially when the electron reservoir is low dimensional. Fortunately, the length of electron reservoirs can now be precisely defined by modern methods used in nanotechnology [25].

To further understand the influence of plasma waves on the device performance, signals with the same frequency of $4.0 \mathrm{THz}$ but with different amplitudes were applied on the device under different DC biases. Simulation results are shown in Figure 4. We find that when the device is unbiased, the device performance shows a nearly linear increase with the amplitude of the applied signals. When DC bias is applied, the device performance for small signals is significantly enhanced, but that for large signals is nearly constant. The performance reduces with the amplitude of the applied signals and then reaches a minimal value before increasing in a manner similar to the unbiased case. The performance enhancement induced by the DC bias implies that plasma waves are easier to be excited on a DC flow. Furthermore, we can find from Figure 4 that the applied signal's amplitudes (DC biases) at the minimal performance points are about $0.4 \mathrm{~V}(0.3 \mathrm{~V}), 0.7 \mathrm{~V}(0.5 \mathrm{~V}), 1.1 \mathrm{~V}(0.8 \mathrm{~V})$, and $1.4 \mathrm{~V}(1.0 \mathrm{~V})$. It is interesting to see that, at these minimal points, the amplitude of each applied signal is only about $\sqrt{2}$ times the corresponding DC bias. Because the amplitude of a sinusoidal signal is also $\sqrt{2}$ times its time-averaged value over every semiperiod, one can expect that the time-averaged

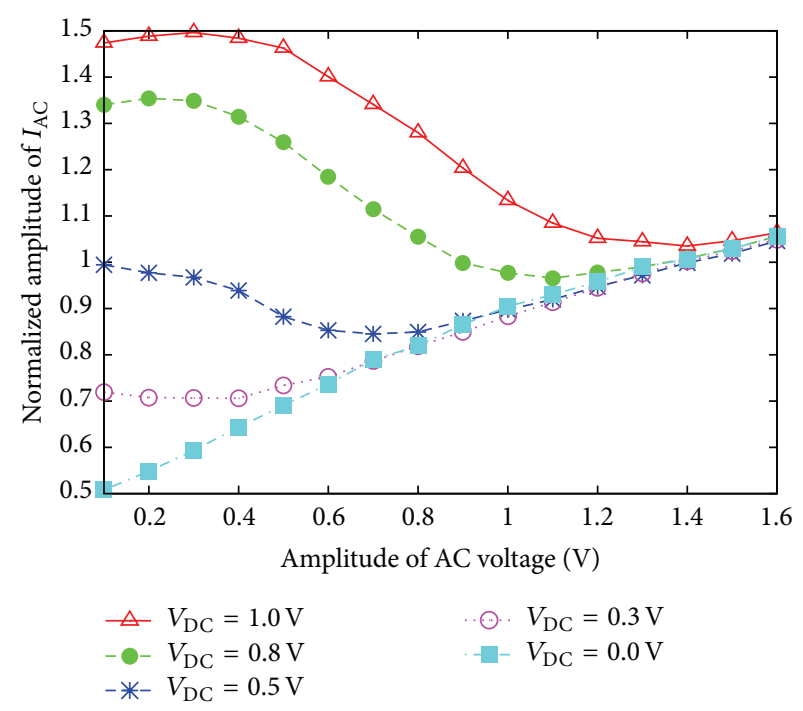

FIGURE 4: Current response of the nanochannel versus the amplitude of applied signal with a frequency of $4.0 \mathrm{THz}$, under different DC voltages of $1.0 \mathrm{~V}$ (red curve), $0.8 \mathrm{~V}$ (green curve), $0.5 \mathrm{~V}$ (navy blue curve), $0.3 \mathrm{~V}$ (pink curve), and $0.0 \mathrm{~V}$ (powder-blue curve).

electric-field should be zero in the device at the negative semiperiod of applied signals. This means that the effect of the DC bias is cancelled when the amplitude of the applied signal is larger than $\sqrt{2}$ times the DC bias; therefore, the device functions as one without any DC bias.

\section{Conclusion}

In this paper, we have employed a combined 2D-3D EMC model to analyse the $\mathrm{THz}$ performances of a $\mathrm{GaN}$-based planar nanochannel device under different conditions. We observed that the performance of the device at frequencies higher than $0.5 \mathrm{THz}$ is strongly enhanced by $2 \mathrm{D}$ plasma waves. The resonances of the $2 \mathrm{D}$ plasma waves with the device structure result in two performance peaks at frequencies of 
about $4 \mathrm{THz}$ and $8 \mathrm{THz}$, respectively. This resonant feature may be applied for frequency selection in RF circuits or $\mathrm{THz}$ systems. Moreover, the application of a DC bias allows for small signals to excite 2D plasma waves easier, resulting in a significant enhancement of the device performance. However, when the applied-signal amplitude is larger than $\sqrt{2}$ times the DC bias, the device operates as one without any DC bias.

\section{Conflict of Interests}

The authors declare that there is no conflict of interests regarding the publication of this paper.

\section{Acknowledgments}

This work was supported by Natural Science Foundation of Guangdong Province, China (no. S2013010012711), NSFC (nos. 11374185, 61072029), and FOK YING TONG Education Foundation (no. 122004). The authors also thank the highperformance computing platform of South China Normal University for technical support.

\section{References}

[1] I. Bisotto, E. S. Kannan, S. Sassine et al., "Microwave based nanogenerator using the ratchet effect in $\mathrm{Si} / \mathrm{SiGe}$ heterostructures," Nanotechnology, vol. 22, no. 24, Article ID 245401, 2011.

[2] H. Irie and R. Sobolewski, "Terahertz electrical response of nanoscale three-branch junctions," Journal of Applied Physics, vol. 107, no. 8, Article ID 084315, 2010.

[3] I. Íñiguez-de-la-Torre, S. Purohit, V. Kaushal et al., "Exploring digital logic design using ballistic deflection transistors through monte carlo simulations," IEEE Transactions on Nanotechnology, vol. 10, no. 6, pp. 1337-1346, 2011.

[4] C. Balocco, S. R. Kasjoo, X. F. Lu et al., "Room-temperature operation of a unipolar nanodiode at Terahertz frequencies," Applied Physics Letters, vol. 98, no. 22, Article ID 223501, 2011.

[5] C. Balocco, M. Halsall, N. Q. Vinh, and A. M. Song, "THz operation of asymmetric-nanochannel devices," Journal of Physics Condensed Matter, vol. 20, no. 38, Article ID 384203, 2008.

[6] J.-F. Millithaler, L. Reggiani, J. Pousset et al., "A Monte Carlo investigation of plasmonic noise in nanometric $n-\mathrm{In}_{0.53} \mathrm{Ga}_{0.47} \mathrm{As}$ channels," Journal of Statistical Mechanics: Theory and Experiment, vol. 2009, no. 1, Article ID P01040, 2009.

[7] M. Dyakonov and M. Shur, "Shallow water analogy for a ballistic field effect transistor: new mechanism of plasma wave generation by dc current," Physical Review Letters, vol. 71, no. 15, pp. 2465-2468, 1993.

[8] A. El Fatimy, N. Dyakonova, Y. Meziani et al., "AlGaN/GaN high electron mobility transistors as a voltage-tunable room temperature Terahertz sources," Journal of Applied Physics, vol. 107, no. 2, Article ID 024504, 2010.

[9] V. Yu. Kachorovskii and M. S. Shur, "Current-induced Terahertz oscillations in plasmonic crystal," Applied Physics Letters, vol. 100, no. 23, Article ID 232108, 2012.

[10] T. Nagatsuma, "Terahertz technologies: present and future," IEICE Electronics Express, vol. 8, no. 14, pp. 1127-1142, 2011.

[11] Y. Chen, J. He, H. L. Liang et al., "Model-based prediction of the plasma oscillation excitation response characteristics of a high-electron mobility transistor-based Terahertz photomixer with the cap region," Journal of Computational and Theoretical Nanoscience, vol. 9, no. 4, pp. 549-554, 2012.

[12] L. Wang, W. Hu, J. Wang et al., "Plasmon resonant excitation in grating-gated AlN barrier transistors at Terahertz frequency," Applied Physics Letters, vol. 100, no. 12, Article ID 123501, 2012.

[13] Y. Zhou, J. Sun, Y. Sun et al., "Characterization of a room temperature Terahertz detector based on a GaN/AlGaN HEMT," Journal of Semiconductors, vol. 32, no. 6, Article ID 064005, 2011.

[14] K. Y. Xu, J. W. Xiong, A. M. Song, and G. Wang, "Effects of three-dimensional electric-field coupling on a side-gated nanotransistor," Semiconductor Science and Technology, vol. 26, no. 9, Article ID 095026, 2011.

[15] I. Iñiguez-de-la-Torre, J. Mateos, D. Pardo, A. M. Song, and T. González, "Noise and Terahertz rectification linked by geometry in planar asymmetric nanodiodes," Applied Physics Letters, vol. 94, no. 9, Article ID 093512, 2009.

[16] K. Y. Xu, X. F. Lu, G. Wang, and A. M. Song, "Strong spatial dependence of electron velocity, density, and intervalley scattering in an asymmetric nanodevice in the nonlinear transport regime," IEEE Transactions on Nanotechnology, vol. 7, no. 4, pp. 451-457, 2008.

[17] K. Y. Xu, G. Wang, and A. M. Song, "Gunn oscillations in a self-switching nanodiode," Applied Physics Letters, vol. 93, no. 23, Article ID 233506, 2008.

[18] T. Sadi, F. Dessenne, and J.-L. Thobel, "Three-dimensional Monte Carlo study of three-terminal junctions based on InGaAs/InAlAs heterostructures," Journal of Applied Physics, vol. 105, no. 5, Article ID 053707, 2009.

[19] T. Sadi and J.-L. Thobel, "Analysis of the high-frequency performance of InGaAs/InAlAs nanojunctions using a threedimensional Monte Carlo simulator," Journal of Applied Physics, vol. 106, no. 8, Article ID 083709, 2009.

[20] I. Iñiguez-de-la-Torre, T. González, D. Pardo et al., “Threeterminal junctions operating as mixers, frequency doublers and detectors: a broad-band frequency numerical and experimental study at room temperature," Semiconductor Science and Technology, vol. 25, no. 12, Article ID 125013, 2010.

[21] A. Íñiguez-de-la-Torre, I. Íñiguez-de-la-Torre, J. Mateos et al., "Searching for THz Gunn oscillations in GaN planar nanodiodes," Journal of Applied Physics, vol. 111, no. 11, Article ID 113705, 2012.

[22] J.-F. Millithaler, I. Iñiguez-de-la-Torre, A. Iñiguez-de-la-Torre et al., "Optimized V-shape design of $\mathrm{GaN}$ nanodiodes for the generation of Gunn oscillations," Applied Physics Letters, vol. 104, no. 7, Article ID 073509, 2014.

[23] K. Y. Xu, Z. N. Wang, Y. N. Wang, J. W. Xiong, and G. Wang, "Influence of electric field coupling model on the simulated performances of a GaN based planar nanodevice," Journal of Nanomaterials, vol. 2013, Article ID 124354, 7 pages, 2013.

[24] M. Shur, "Plasma wave Terahertz electronics," Electronics Letters, vol. 46, no. 26, pp. S18-S21, 2010.

[25] A. Khalid, C. Li, V. Papageogiou et al., " $\operatorname{In}_{0.53} \mathrm{Ga}_{0.47}$ As planar gunn diodes operating at a fundamental frequency of $164 \mathrm{GHz}$," IEEE Electron Devices Letters, vol. 34, no. 1, pp. 39-41, 2013. 

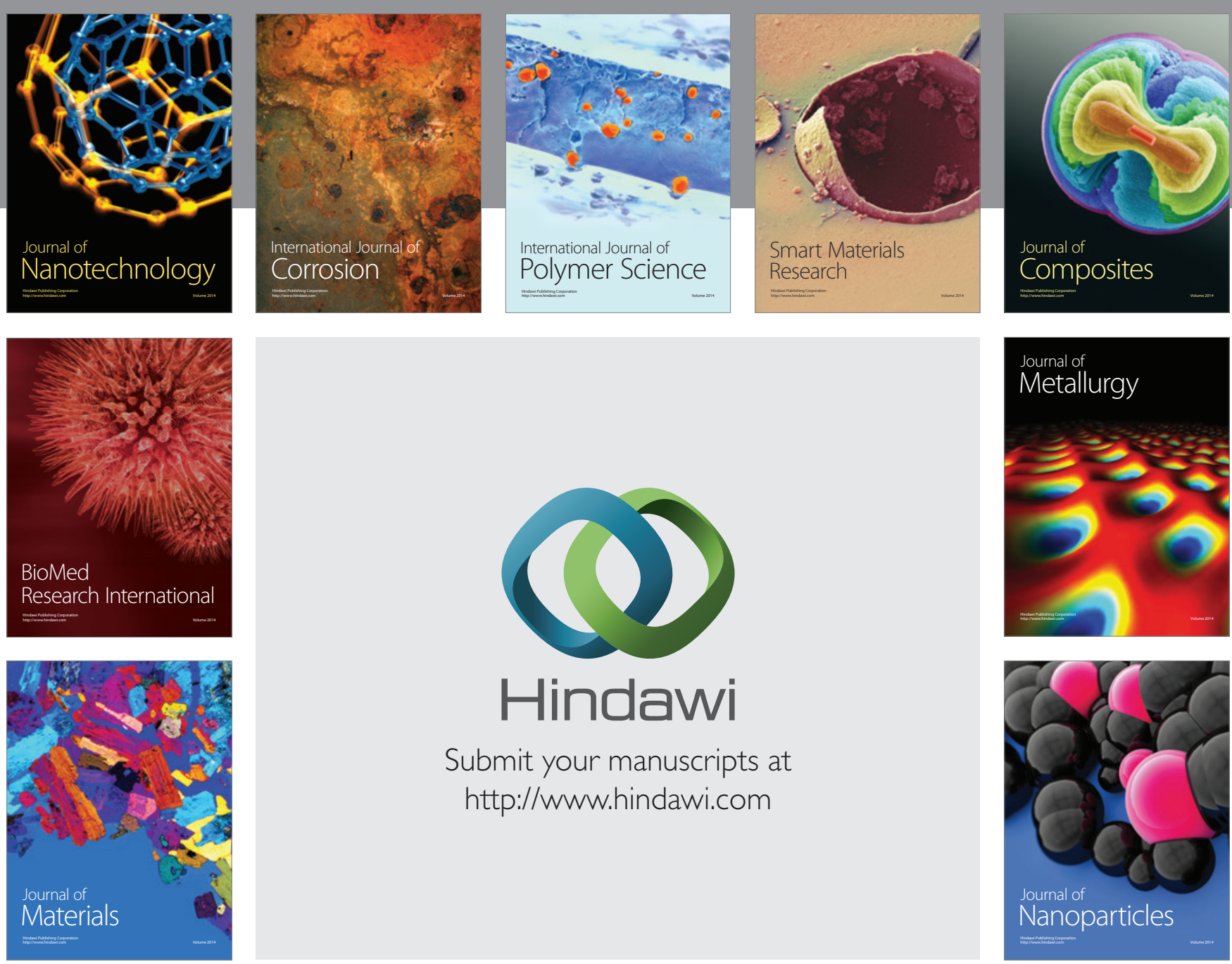

Submit your manuscripts at http://www.hindawi.com
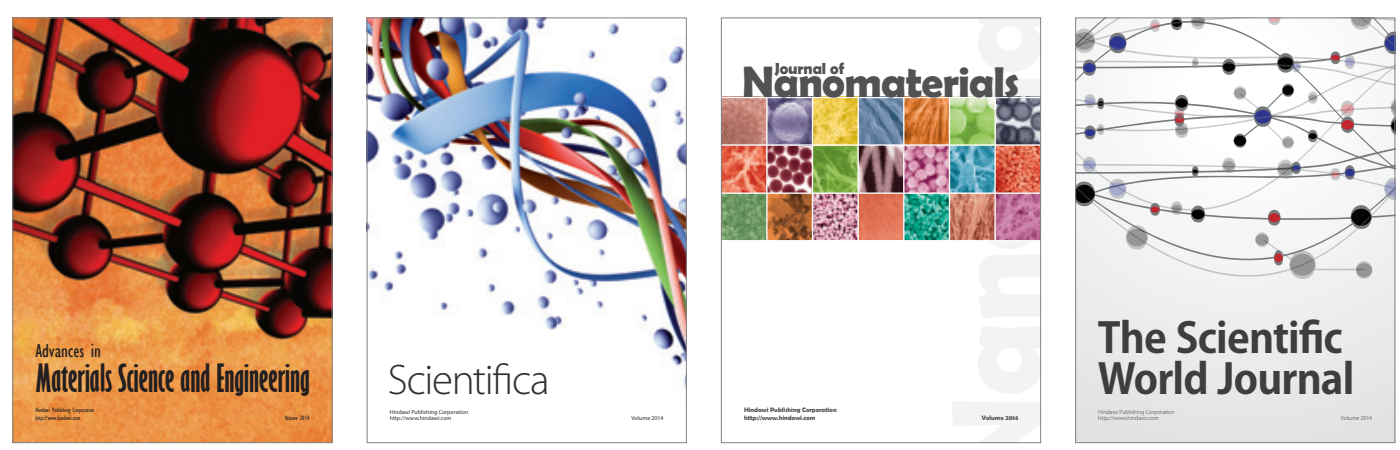

\section{The Scientific World Journal}
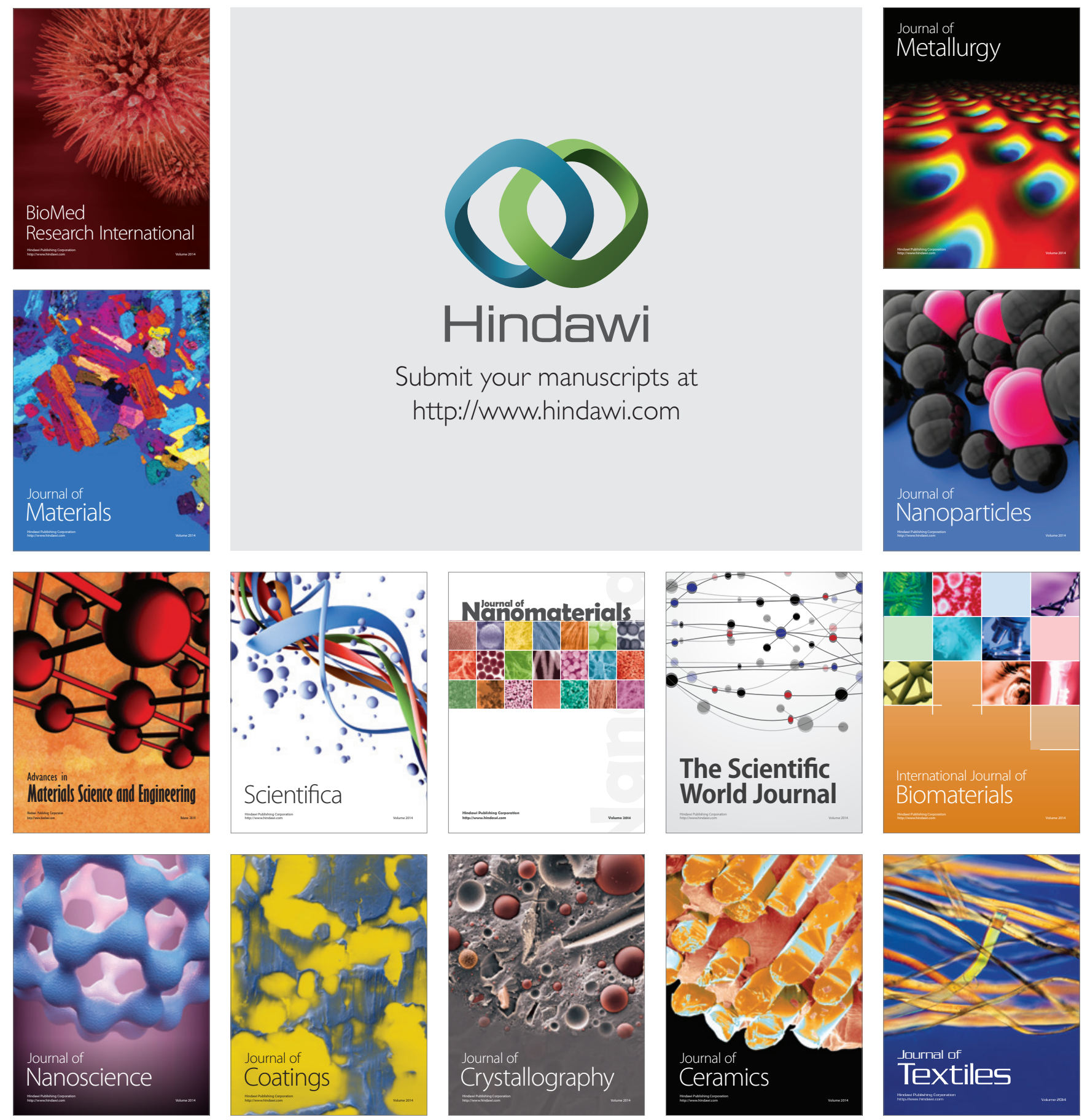